\title{
EL ACCIDENTE DE CHERNÓBIL
}

\section{THE CHERNOBYL ACCIDENT}

César Sánchez: Central Nuclear de Trillo. Guadalajara (España) cesarsual@terra.es

\section{CURRÍCULUM VITAE}

Ingeniero operador de la Central Nuclear de Trillo, en Guadalajara (España).

\section{RESUMEN}

El año 2006 nos dejó, entre otras cosas, el XX aniversario del accidente de Chernóbil. Chernóbil es en la actualidad una ciudad fantasma situada al norte de Ucrania, cerca de la frontera con Bielorrusia, después de quedar abandonada por el accidente ocurrido en la central nuclear que llevaba el mismo nombre el 26 de abril de 1986, mientras se experimentaba con el cuarto reactor para comprobar si la energía de las turbinas podía generar suficiente electricidad para las bombas de refrigeración en caso de fallo. Una sucesión de errores provocó una enorme subida de potencia y una gran explosión que dejó al descubierto el núcleo del reactor. Éste emitió una gigantesca nube radiactiva hacia toda Europa. Cómo funcionan los reactores, su comportamiento, una descripción detallada del accidente y sus consecuencias se pueden encontrar en este texto. 
Chernóbil - Accidente - Central Nuclear - Reactor

\begin{abstract}
In 2006 he left us, among other things, the twentieth anniversary of the Chernobyl accident. Chernobyl is now a ghost town located in northern Ukraine, near the border with Belarus, after being abandoned by the accident at the nuclear plant bearing the same name on April 26, 1986, while experimenting with the fourth reactor to check if the power of the turbines could generate enough electricity for cooling pumps in case of failure. A series of errors led to a huge increase in power and a large explosion that exposed the reactor core. This gave a huge radioactive cloud into Europe. How the reactors, their behavior, a detailed description of the accident and its consequences can be found in this text.
\end{abstract}

\title{
KEY WORDS
}

Chernobyl - Accident - Nuclear Power Station - Reactor

\section{ÍNDICE}

\section{Introducción}

2. Los reactores nucleares RMBK

3. Descripción del accidente

4. Responsables de la catástrofe

5. Consecuencias inmediatas del accidente

6. Conclusión

7. Notas de VA 


\section{TEXTO:}

\section{Introducción}

Una vez terminado el 2006, vigésimo cumpleaños del desastre de Chernobyl y ya cesado, desde hace algunos meses, el bombardeo informativo recordando este hecho, veo con resignación que, al igual que en los diecinueve años anteriores, nada se ha hablado realmente interesante sobre este accidente, en los diferentes medios de comunicación.

Viendo este desolador panorama y harto de mentiras y sensacionalismos, voy a intentar contar qué fue lo que realmente pasó, y lo voy a hacer de la manera más simple y aséptica posible, de forma que todo el mundo lo pueda entender.

Espero de aquellos que conocen bien el tema su perdón, por no entrar del todo a fondo o, si en algún momento, alguna cosa no está bien explicada.

Sin más preámbulos pasemos a la cuestión.

\section{Los reactores nucleares $\mathrm{RMBK}$}

Chernobyl es la ciudad que da nombre a la "Central eléctrica nuclear memorial V.I. Lenin", a pesar de estar alejada $18 \mathrm{Km}$ de ella y de haber otras ciudades más cerca. Está situada a unos $160 \mathrm{Km}$ al nordeste de Kiev y vive del comercio y la industria. En el momento del accidente tenía unos 44.000 habitantes

Prypjat nació, sin embargo, a la sombra de la central. Creada en 1970 para que vivieran los trabajadores de la central y sus familias, posee una posición geográfica estratégica, disfruta de un clima relativamente templado y tiene un suelo muy fértil. 
Todo esto hizo que tuviese un rápido desarrollo, llegando a tener, en el momento del accidente, una población entorno a las 50.000 personas.

La "Central eléctrica nuclear memorial V.I. Lenin" (V.I. son las iniciales de Vladimir Ilich) estaba formada por cuatro obsoletos reactores tipo RBMK 1000 de 3.200 Mw de potencia térmica y 1.000 Mw de potencia eléctrica y tenía prevista la construcción de otros dos más del mismo tipoNOTA_1.

RBMK son las iniciales de Reaktor Bolshoy Moshchnosti Kanalniy que más o menos significa "reactor de gran potencia de tipo canal". Hay que aclarar que no se diseñó para la producción de energía eléctrica, éste es un uso secundario, sino para la obtención de plutonio para armas nucleares.

Estos reactores usan como combustible Uranio 238 con muy bajo enriquecimiento, entorno al $2 \%$ de Uranio 235 y son del tipo de agua ligera en ebullición moderados por grafito. Es decir, la refrigeración de los elementos combustibles se realiza con agua ligera, que es el agua normal y corriente, eso sí, depurada. El agua entra en forma líquida por la parte inferior del núcleo a través de cada canal y va refrigerando al combustible, mientras ella se calienta, llegando a pasar a ser vapor en la parte superior del mismo. Este vapor es el que se envía a las turbinas para producir energía eléctrica. A su vez, la moderación neutrónica es realizada, básicamente, por el grafito constituyente del núcleo.

Los neutrones creados en las fisiones nucleares nacen con energías muy elevadas, sin embargo, sólo a energías muy bajas producen la fisión del uranio, por lo que es necesario que pierdan la mayor parte de su energía. Esto se consigue mediante choques con los átomos del moderador y a este proceso se le llama moderación. 
El núcleo de estos reactores está formado, básicamente, por un cilindro de grafito de unos $7 \mathrm{~m}$ de altura y $12 \mathrm{~m}$ de diámetro, en el que hay practicados en torno a 1.700 canales, en 1.600 de los cuales, aproximadamente, se alojan los elementos combustibles y las barras de control quedando el resto para los demás elementos necesarios para el funcionamiento del reactor.

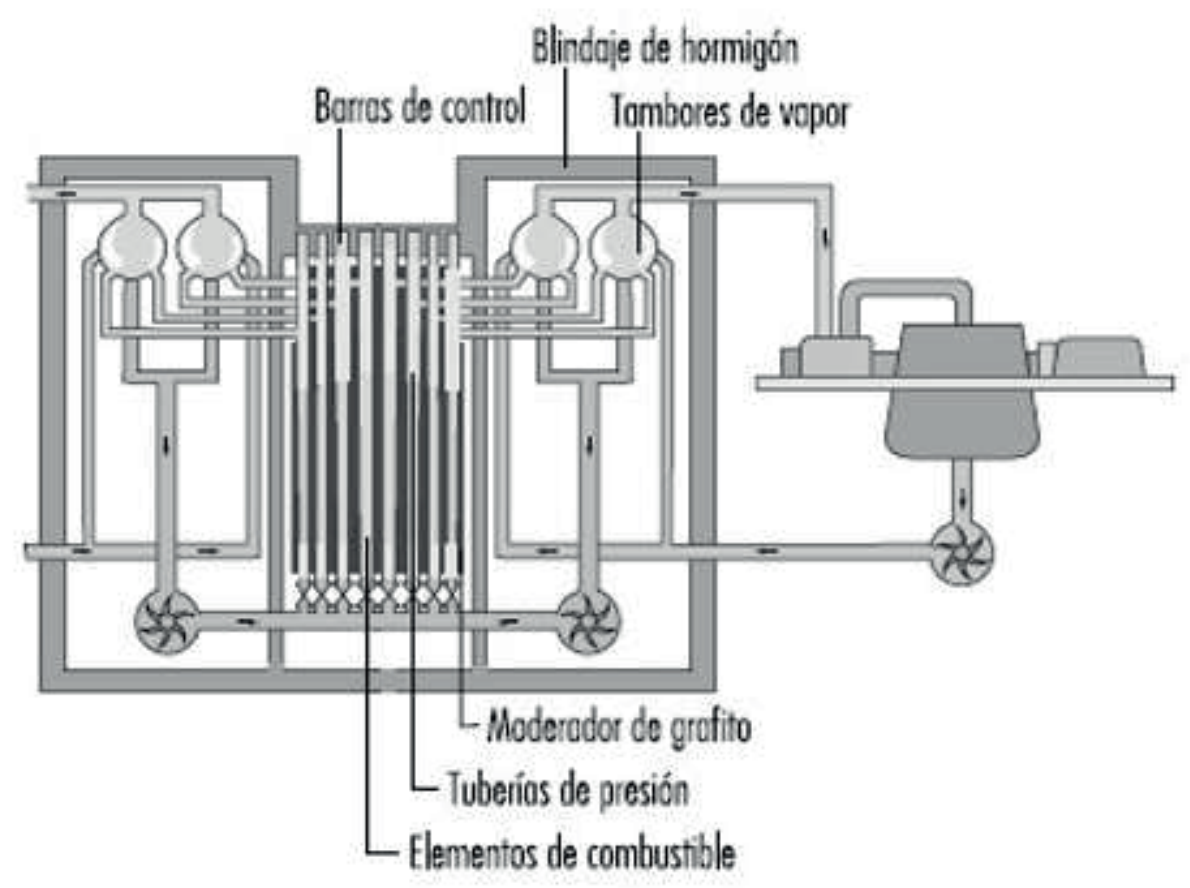

\section{Reactor de ogua ligero en ebullición moderedo con grufito (RBMK)}

Para evitar la fuga neutrónica, el núcleo se rodea de una fuerte pantalla que mantiene a los neutrones confinados en él.

Por otra parte, la temperatura del núcleo sube a medida que aumenta la potencia, por lo que, en operación a potencia nominal, la temperatura del grafito es muy elevada y éste tiene la mala costumbre de arder a altas temperaturas, si se encuentra con oxigeno. A causa de esto, todo el núcleo se cubre de una estructura metálica sellada en la que se introduce un gas inerte para evitar que el aire entre en contacto con el grafito. 

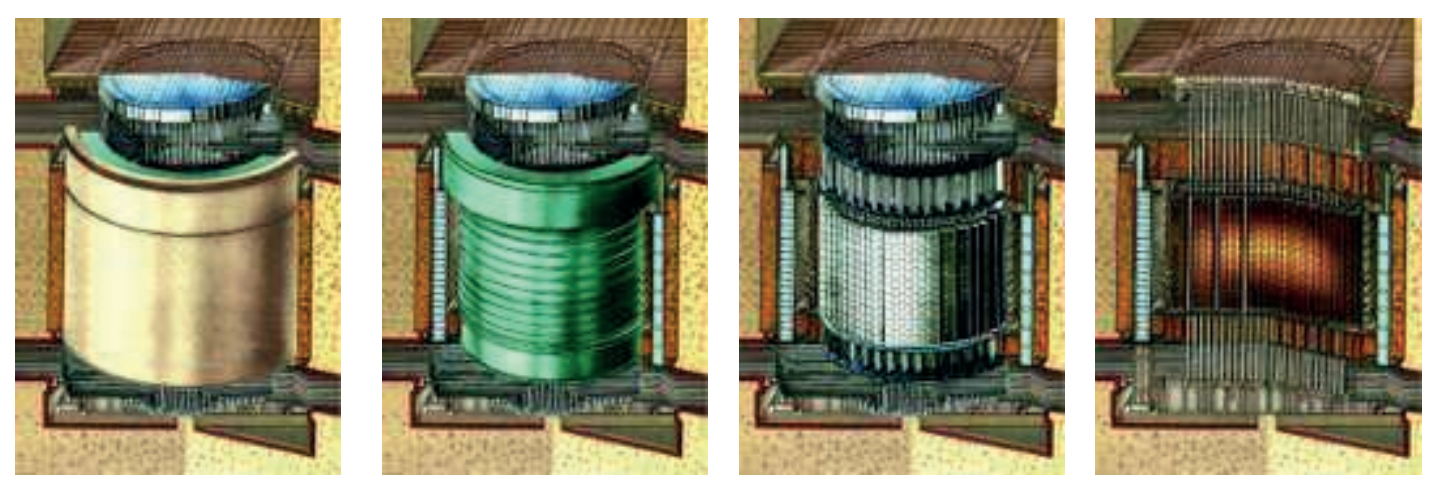

Además, como estos reactores se diseñaron para la producción de plutonio militar y la fabricación de bombas es continua, tienen la posibilidad de extraer los elementos combustibles sobre la marcha, sin necesidad de parar el reactor. Esto obliga a grandes estructuras de blindaje y a robustos y abundantes equipos de maniobra situados encima del núcleo.

Todo ello hace que el edificio que alberga al reactor tenga un tamaño muy grande, de más de 70 m de altura, y la construcción de un resistente edificio de contención es muy costosa, además de entrañar ciertas dificultades constructivas. 


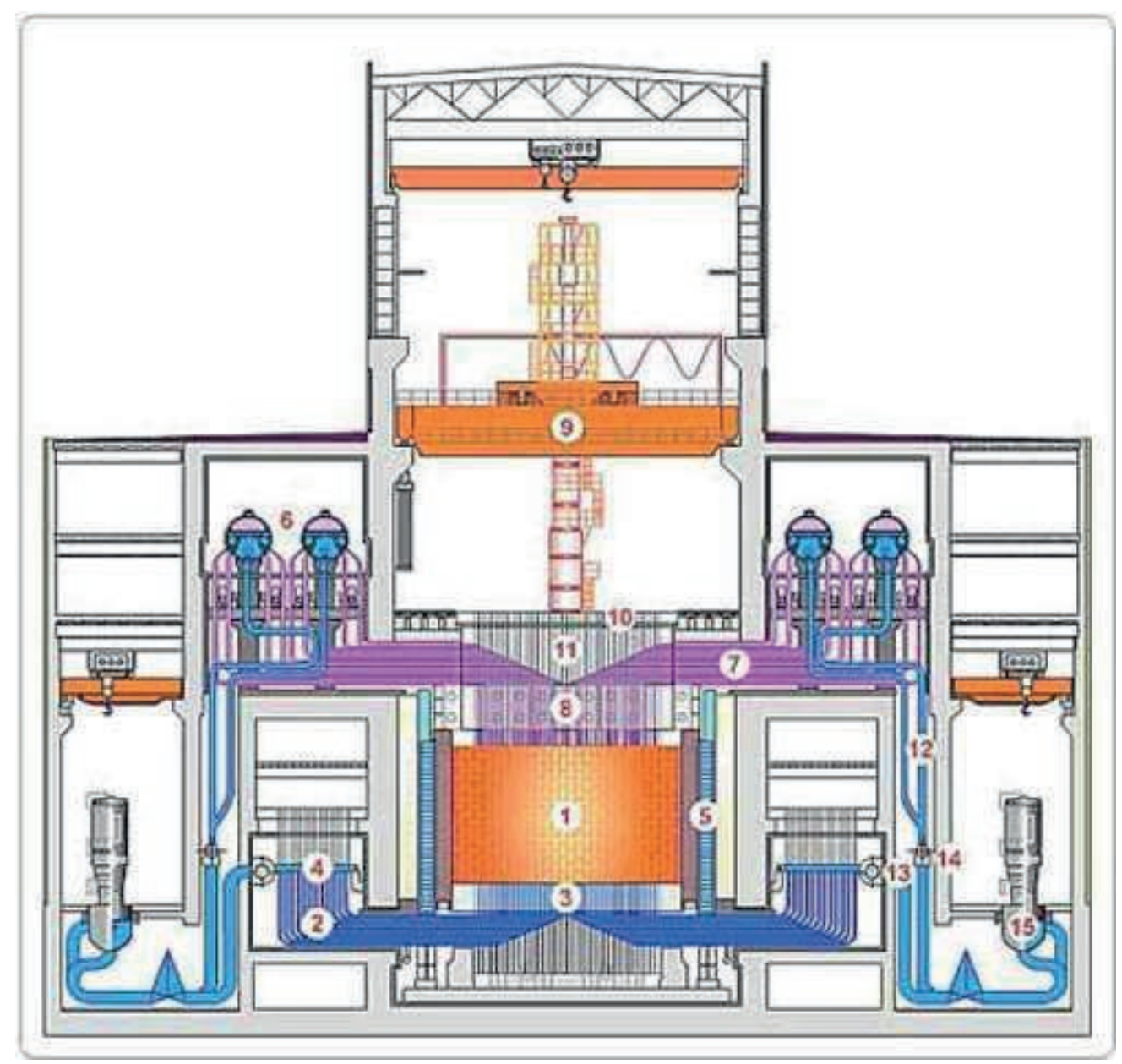

La potencia de un reactor nuclear es función directa de la población neutrónica y ésta varía en función de la reactividad. Si la reactividad es positiva la población neutrónica aumenta, lo que hace aumentar, a su vez, la potencia $\mathrm{y}$, en consecuencia, la temperatura. Si la reactividad disminuye, el proceso es el inverso y, si la reactividad es cero, la población neutrónica permanece constante en el tiempo.

En los reactores nucleares, la reactividad es ligeramente positiva hasta alcanzar la potencia nominal, es cero durante la operación estable a potencia nominal y es negativa cuando se desea bajar potencia.

Se denominan Coeficientes Inherentes de Reactividad a aquellos que se derivan de las cualidades físicas intrínsecas de los materiales constituyentes del reactor. 
Estos coeficientes influyen en la reactividad y son:

Quemado y transformación del combustible.

Coeficiente de temperatura del moderador.

Coeficiente de huecos.

Coeficiente de temperatura del combustible.

Defecto de potencia.

Redistribución.

Coeficientes de reactividad aproximados de un reactor $\mathrm{RBMK}\left(\mathrm{Dr} \% /{ }^{\circ} \mathrm{C}\right)$

Coeficiente de temperatura de combustible - 0,00125

Coeficiente de potencia a potencia nominal - 0,000005

Coeficiente de temperatura del moderador (grafito) $+0,0006$

Coeficiente de huecos $\quad+0,002$

Estos reactores son estructuralmente inestables debido a que los coeficientes de moderador y de huecos son positivos y con valores tan elevados, respecto de los otros dos, sobre todo el de huecos, que hacen que el coeficiente total sea positivo. Es decir, que, si se produce un aumento de potencia, la cinética del reactor hace que a este aumento siga otro y así sucesivamente. De igual forma, en caso de una bajada de potencia, ésta seguiría bajando. En un reactor estable, una variación de la potencia provoca unas reacciones que hacen que ésta tienda a estabilizarse en un punto próximo al que tenía.

Este comportamiento se debe al diseño de este tipo de reactores.

El coeficiente de temperatura del combustible es debido al llamado efecto Doppler. Este consiste en lo siguiente: las bandas de absorción de neutrones (la energía a que los neutrones son absorbidos y retirados del proceso) se ensanchan al aumentar la 
temperatura del combustible. Es decir, cuando el combustible se calienta la absorción de los neutrones no productores de fisiones aumenta y, en consecuencia, éstas disminuyen, por lo que, si no se toman ciertas medidas, la potencia baja.

En cada canal hay un elemento combustible formado por una serie de varillas, en cuyo interior están alojadas las pastillas de uranio. Tanto a través del elemento como entre éste y el grafito, circula el refrigerante.

A las burbujas de vapor que aparecen en el agua cuando llega a la saturación se las llama huecos, y a estos huecos es a lo que se refiere el coeficiente del mismo nombre.

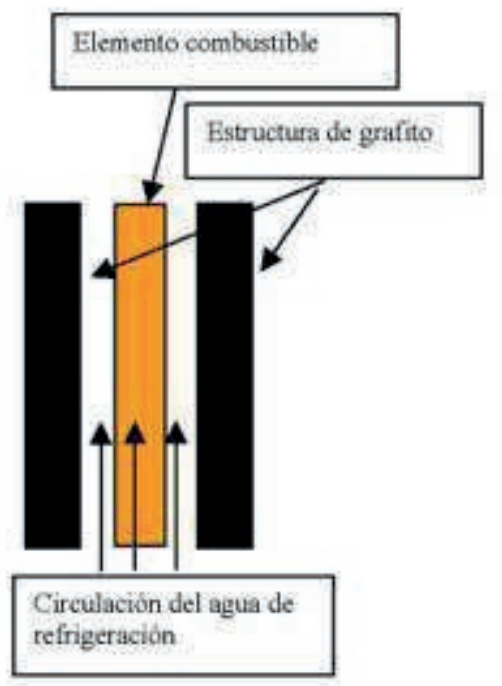

Como se dijo más arriba, los neutrones de muy alta energía (se les llama genéricamente neutrones rápidos) nacen en el combustible y se moderan, casi en su totalidad, en el grafito. Una vez moderados, reciben el nombre de neutrones térmicos, pues su energía se corresponde con la temperatura del moderador. Para estos neutrones térmicos, el agua líquida situada entre el elemento combustible y el grafito actúa de pantalla. 
Por otra parte, hay una pequeña fracción de neutrones que se también se moderan en el agua que refrigera al combustible y vuelven a éste produciendo fisiones.

En un reactor RBMK sobremoderado domina el primer efecto con lo cual, si nos quedamos sin refrigerante, aumenta la reactividad. Esta es la causa del coeficiente de huecos positivo.

En un reactor RBMK submoderado domina el segundo efecto por lo que, si eliminamos el agua de refrigeración, estaremos disminuyendo la reactividad.

Para corregir estas y otros tipos de inestabilidades que presentan los reactores RBMK, éstos deben operarse con, al menos, 30 barras de control introducidasNOTA_2, además de ciertas configuraciones termohidráulicas como es, entre otras, la relación entre el caudal de recirculación de refrigerante a través del núcleo y la cantidad de vapor producido.

Con la introducción de estas 30 barras se pretende que el conjunto de los coeficientes inherentes de reactividad sea negativo.

Los cambios en la relación entre el caudal de agua en recirculación y la cantidad de agua convertida en vapor, permite evitar que el reactor esté en régimen de sobremoderación.

Por otra parte, y dentro de los análisis de accidentes, ante la posibilidad de que éstos pueden ocurrir y para evitar altos niveles de energía en el inicio de los mismos, los sistemas de protección detienen al reactor instantáneamente y de manera automática, en caso de alcanzar determinados niveles de presión, temperatura, caudal, 
disposiciones operativas etc. Esta parada se realiza introduciendo las barras de control.

Para asegurar la parada del reactor, se tienen extraídas un número de barras suficiente que, en caso necesario, son introducidas rápidamente en el núcleo. En los reactores occidentales el tiempo para que se inserten las barras en su totalidad es inferior a $2 \mathrm{~s}$, sin embargo, en los reactores RBMK esta inserción dura $20 \mathrm{~s}$, tarda en ser efectiva unos cuantos segundos más, en torno a $10 \mathrm{~s}$; y además, para terminar de empeorar las cosas, durante los primeros 2 ó 3 segundos de la inserción se mejora la moderación lo hace que la potencia aumente, si no se toma algún tipo adicional de precaución.

Una característica de todos los reactores nucleares es que, una vez que se paran, es decir, se han introducido todas las barras de control, se sigue produciendo calor pues sigue habiendo fisiones debido a neutrones térmicos que nacen en las barras de combustible e interaccionan inmediatamente con átomos de combustible vecinos. Es lo que se llama calor residual y constituye el objetivo principal de los operadores de centrales nucleares, una vez parado el reactor, pues éste alcanza valores de temperatura nada detestables en los primeros minutos de la operación. 


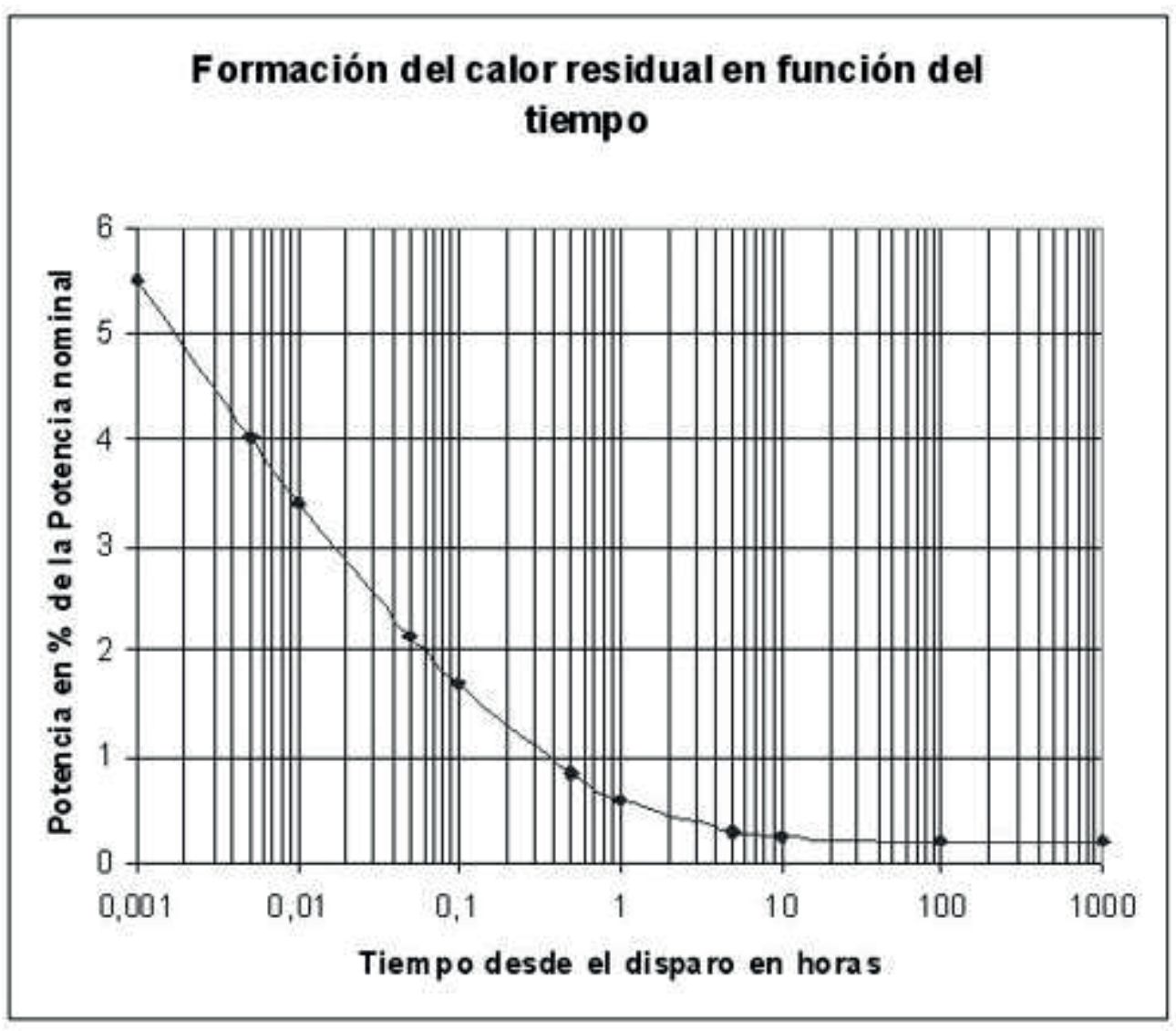

Cuando el reactor está funcionando, se desvía una pequeña parte de la energía eléctrica producida por el turbogrupo para alimentar los sistemas necesarios para el correcto funcionamiento de la planta. Cuando el reactor se para, esta energía eléctrica se toma de la red exterior. Por esta razón es necesario contar con sistemas de seguridad que aseguren la refrigeración del núcleo del reactor una vez parado éste y en caso de pérdida de alimentación eléctrica desde el exterior.

Precisamente durante la madrugada del 26 de abril de 1.986 estaba previsto realizar una prueba en la unidad número cuatro, la más nueva de la central de Cernobyl, consistente en ver la capacidad de refrigeración del núcleo, en caso de pérdida del suministro energía eléctrica desde la red exterior. 
Esta prueba consistía en alimentar 4 de las 8 bombas de recirculación de agua de refrigeración, con la energía que aún se produciría durante bajada de revoluciones del turbogrupo, una vez que no llegara vapor a la turbina, y se realizaría entre 700 y $1.000 \mathrm{Mw}$ de potencia térmica.

Al iniciar la bajada de potencia hasta el nivel deseado, el equivalente a nuestro despacho de carga, obliga a detenerla durante unas $9 \mathrm{~h}$ lo que hace que la concentración de Xenón aumente por encima de lo previsto. El Xenón es un gas cuya característica principal en un reactor es el ser un poderoso absorbente de neutrones.

\section{Descripción del accidente}

Una vez obtenido el permiso para iniciar la bajada de potencia hasta el nivel de la prueba, ésta cayó hasta, aproximadamente, $30 \mathrm{Mw}$ térmicos. No está claro si a causa de un fallo en el control automático de la potencia o del transitorio del Xenón. Después de unas dos horas se consiguió estabilizar el reactor a una potencia de 200 Mw térmicos.

Esta bajada de potencia hizo que subiera aún más la concentración de Xenón y, para evitar que el reactor se apagara se extrajeron barras de control más allá de lo permitido, dejando introducidas sólo 8 de las 30 que se necesitaban. 


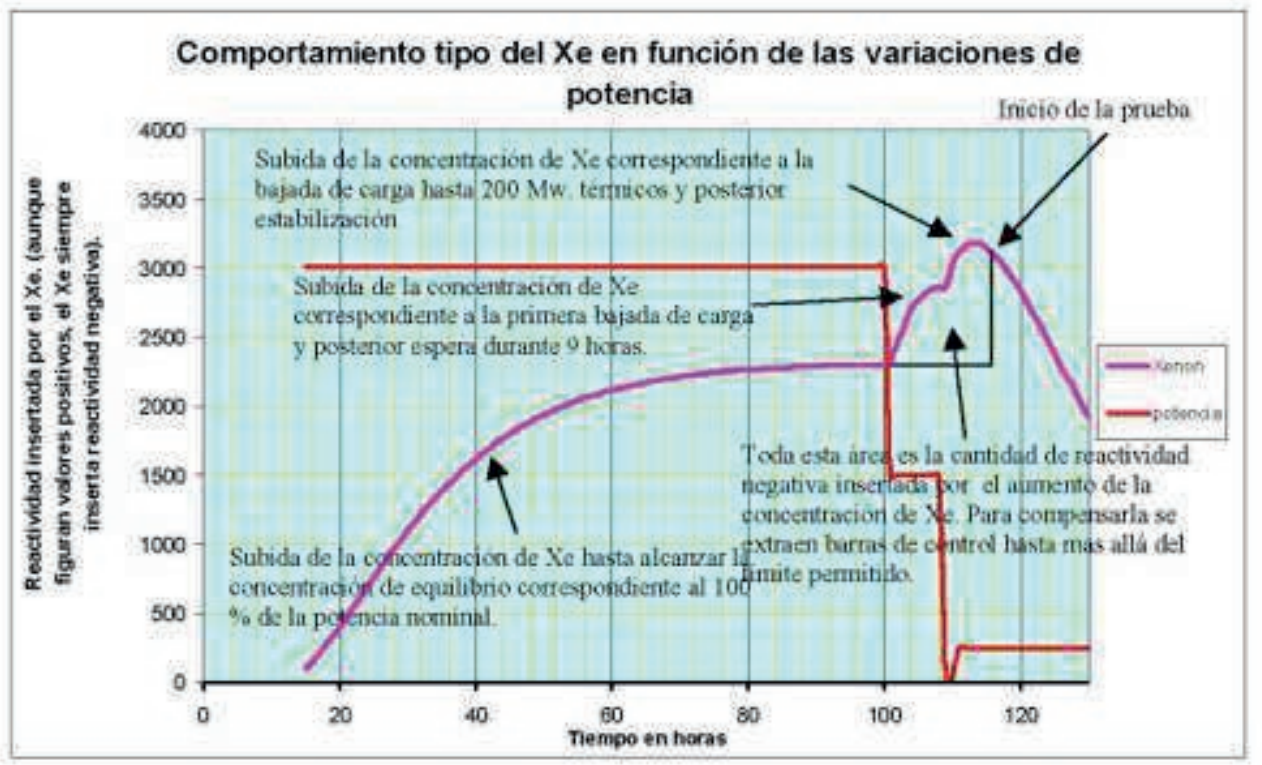

En esta figura se refleja un ejemplo del comportamiento típico del Xe correspondiente a la operación antes descrita. Tanto los valores como el momento de inicio de la prueba son sólo orientativos.

La disposición de equipos para la prueba obligaba a bloquear el disparo del reactor. Así mismo, para observar la capacidad de refrigeración, que era el motivo de la prueba, se bloqueron los equipos de refrigeración de emergencia.

El estado termohidráulico de la planta antes del experimento era muy diferente del nominal. El flujo de recirculación del refrigerante era muy alto y la producción de vapor proporcionalmente muy pequeña. La presión del primario era también inferior a la nominalNOTA_3. La temperatura del refrigerante a la entrada del reactor era muy próxima a la de ebullición. En la salida, la calidad del vapor era muy pobre, por el alto caudal de recirculación. En ese momento el reactor se encontraba en una situación intrínsecamente inestable.

Siempre se debe de operar una central nuclear manteniendo los siguientes Objetivos de Protección: 
Asegurar la subcriticidad: Es decir, asegurar la parada del reactor. Este objetivo no se cumplía en ese momento, al tener bloqueados los disparos automáticos, al grado de inestabilidad existente y a la lentitud de entrada de las barras de control.

Asegurar la refrigeración del núcleo. Este objetivo no se cumplía durante la prueba, al tener desconectados los sistemas de refrigeración.

Asegurar el confinamiento de la radiactividad. Este objetivo no se cumplía pues no existía edificio de contención.

Es decir, en el momento de la prueba no se cumplía ninguno de los Objetivos de Protección.

26 de Abril de 1986. - 01 h. 23 min. 04 s. En este momento, con un reactor inestable y con los elementos de seguridad bloqueados se inicia la prueba.

(Es como si a un camión le anulamos los frenos y bloqueamos la dirección y lo soltamos por una cuesta abajo)

Para realizar la prueba programada, con cuatro de las ocho bombas de recirculación del refrigerante paradas, se procede al cierre de las válvulas de vapor de la admisión del único turboalternador que estaba funcionando. (Estas maniobras hubieran disparado el reactor si la protección automática no hubiera estado bloqueada ).

Las cuatro bombas restantes, alimentadas sólo con la electricidad producida durante la parada del alternador, no son capaces de refrigerar al núcleo del reactor y, como estaban bloqueados, no entran en funcionamiento los equipos de refrigeración de emergencia. 
La temperatura del refrigerante subió y empezó a hervir. A causa del coeficiente positivo de huecos, se introdujo una reactividad positiva elevada que hizo a la potencia multiplicarse por cien en sólo unos segundos. Debido al coeficiente negativo del combustible (efecto Doppler), se compensó este aumento de potencia, al insertarse una reactividad negativa que la hizo bajar.

Desafortunadamente, la elevada temperatura que ya tenía el combustible hizo vaporizarse al refrigerante, produciéndose lo que se llama, o llamó: explosión de vapor. Esto hizo que la envolvente del reactor quedara muy dañada y el núcleo seco.

Los operadores procedieron a la parada manual del reactor, pero las barras de control no entraron. Quizás esto fue debido a que ya existía cierta deformación en el núcleo, imposibilitando mecánicamente el deslizamiento de las barras entre la estructura. En cualquier caso, aunque hubieran entrado, hubiera servido de poco debido a su lentitud en insertarse en el núcleo.

Debido al coeficiente positivo de huecos, comenzó a establecerse una reactividad positiva y ésta ya no la puede compensar el efecto Doppler. La potencia del reactor aumentó de manera descomunal en un tiempo muy breve. Se estima que la potencia pudo ser varios miles de veces mayor que la inicial, en sólo unos minutos.

Como la temperatura era tan grande, el núcleo comenzó a fundirse. Uno de los efectos que tiene este fenómeno es la producción de grandes cantidades de hidrógeno.

Llegado un momento, este hidrógeno llegó a alcanzar la concentración suficiente como para provocar una devastadora explosión que destruyó el edificio del reactor, sobre todo, la cubierta que, literalmente, desapareció, e incendió el grafito del núcleo. 


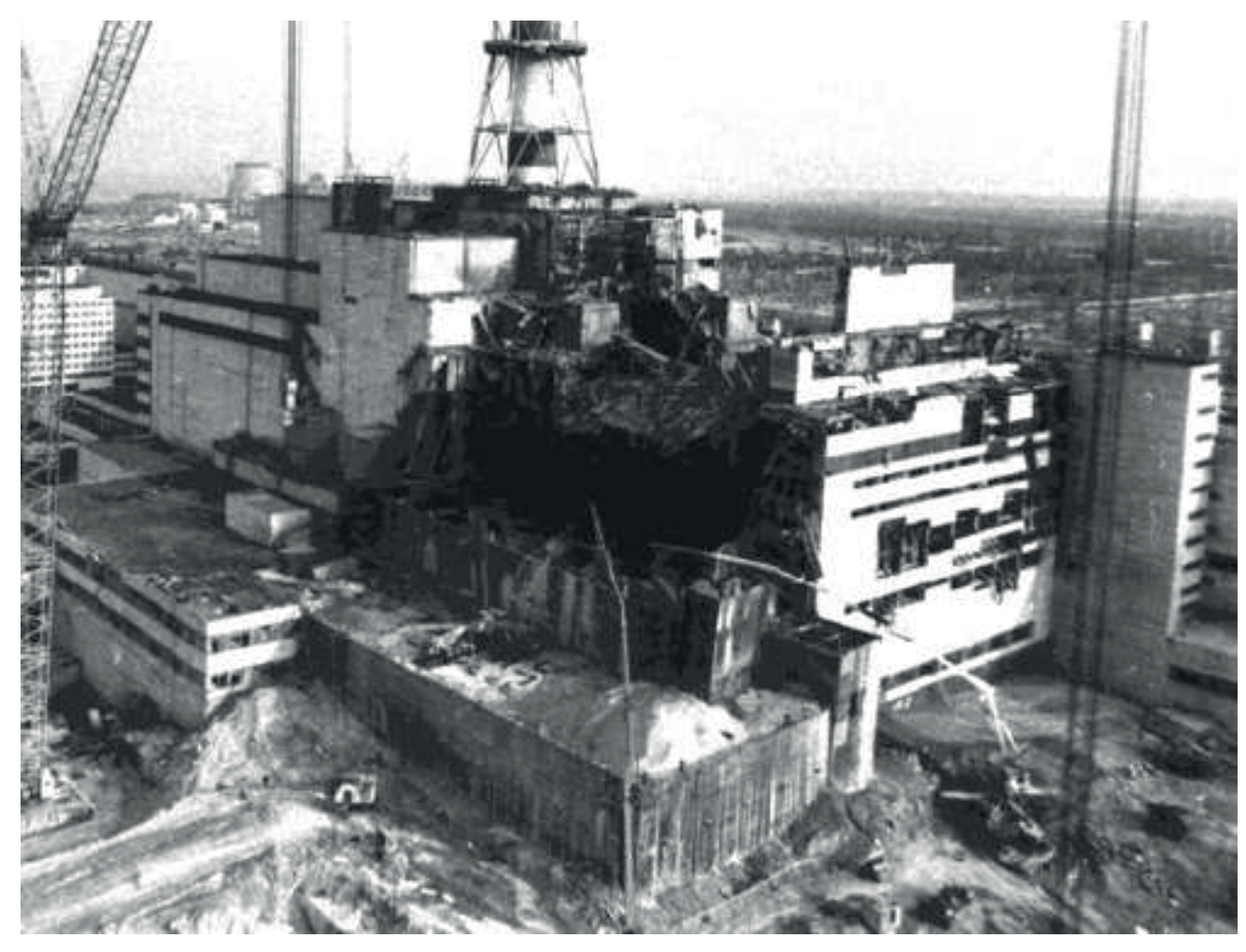




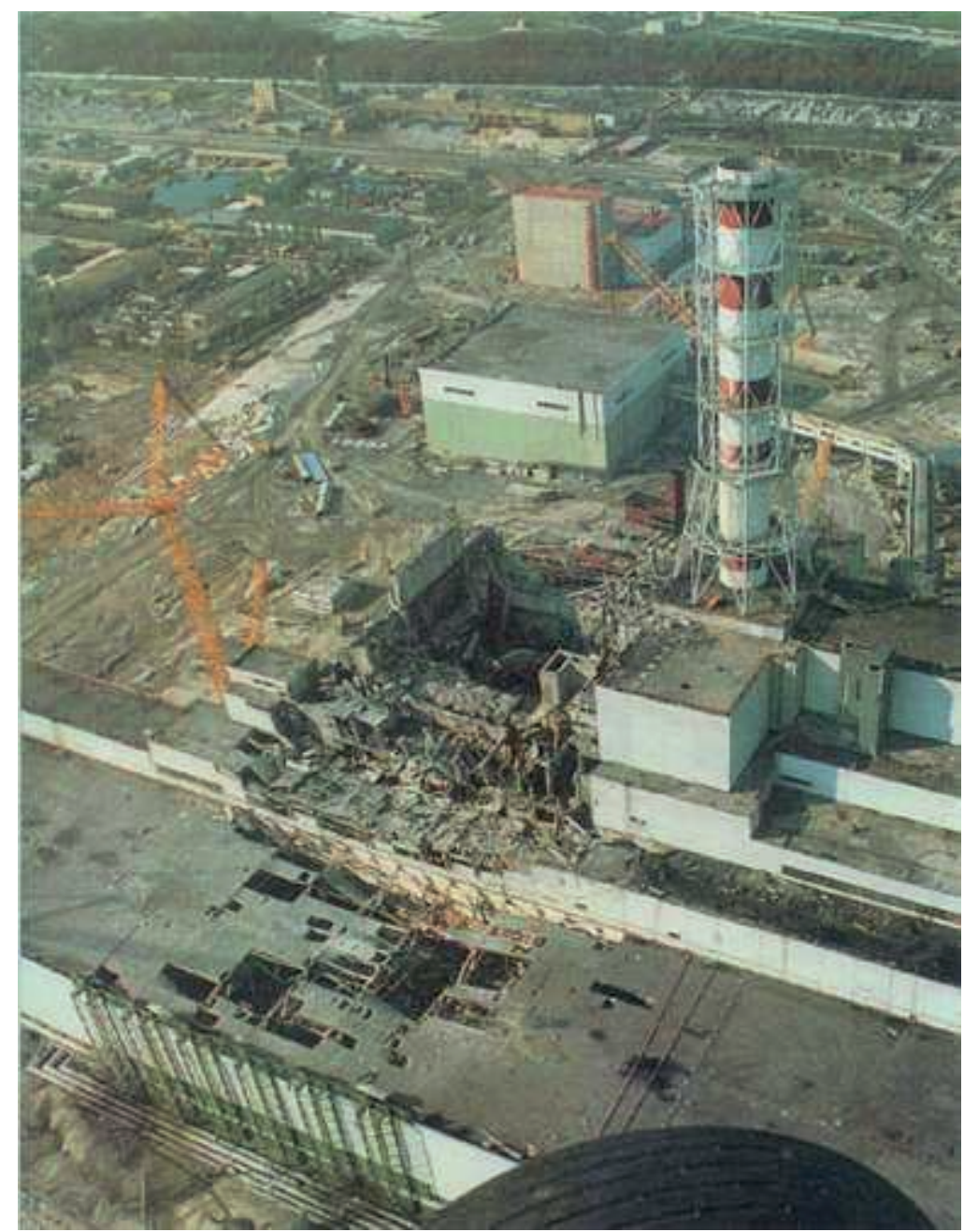

Sólo desde el momento en que comenzó la fusión del núcleo, empezó a pararse el reactor, al perder éste su geometría, pero la producción de calor aún prosiguió.

Las vainas que encierran las pastillas de combustible se destruyeron, por lo que los productos de fisión escaparon libremente a la atmósfera, creándose una gigantesca nube de humo y productos de fisión altamente radiactivos.

El primer acercamiento en helicóptero evidenció la magnitud de lo ocurrido. El núcleo, expuesto a la atmósfera, continuaba ardiendo. La temperatura alcanzó los $2.500{ }^{\circ} \mathrm{C}$ y, en un efecto chimenea, impulsaba el humo radiactivo a una altura considerable. 
El panorama era dantesco. El grafito estaba ardiendo, el combustible fundiéndose y se estaban inyectando en la atmósfera miles de toneladas de productos altamente radiactivos, entre ellos el temido Yodo 131NOTA_4.

Una masa fundente, altamente radiactiva, similar a la de la imagen mostrada a continuación, y formada por la fusión de todos los materiales constitutivos del núcleo, llamada corium o lava de combustible, funde todo lo que encuentra a su paso.
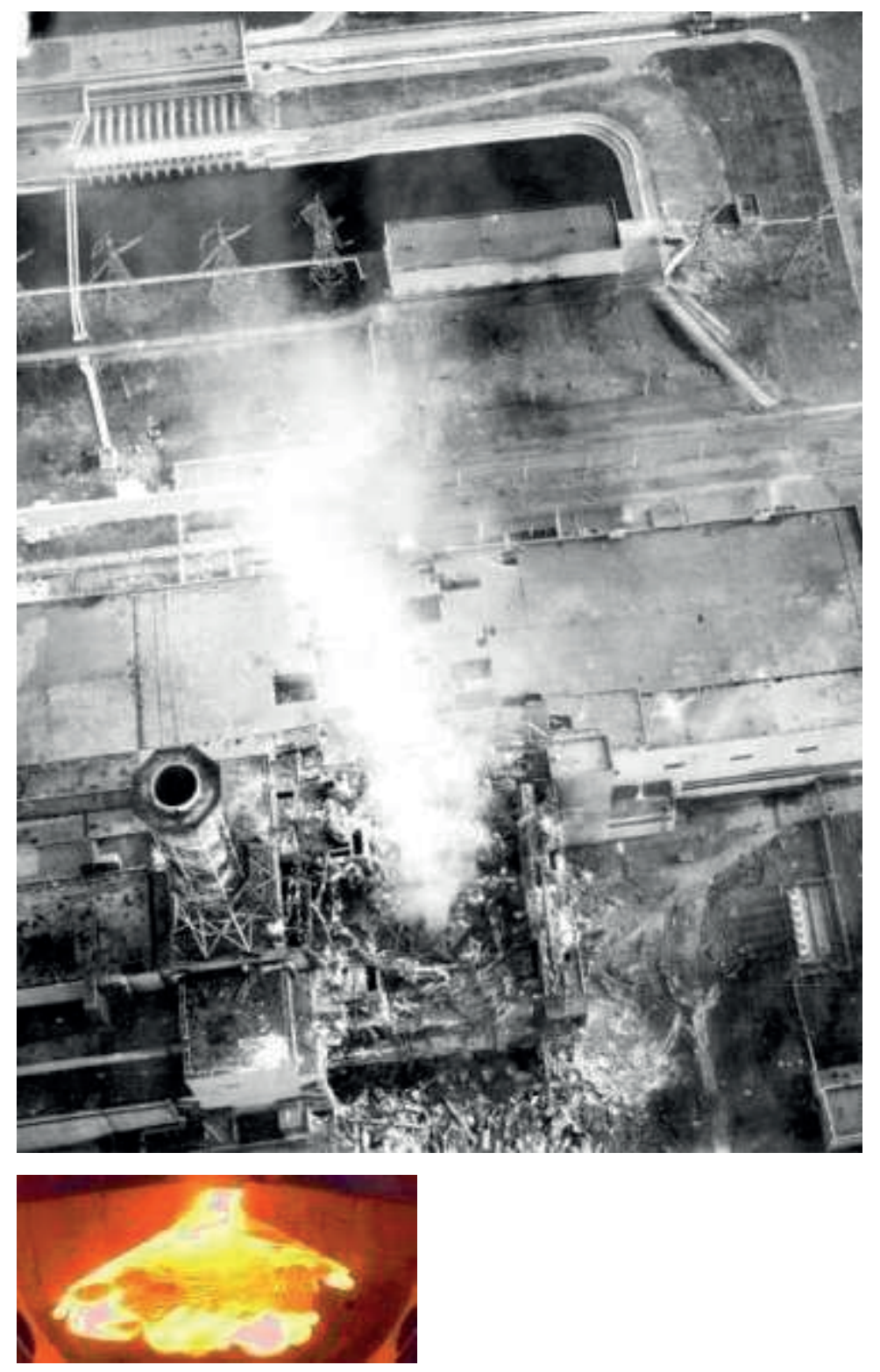
Esta masa incandescente penetró en los cubículos existentes en la parte inferior del reactor solidificándose a medida que se iba enfriando y dejando grandes masas de corium radiactivo. Por otro lado, la explosión de Hidrógeno hizo que parte del núcleo se desintegrara.

Minutos después del accidente, todos los bomberos militares asignados a la central, ya estaban en camino. Las llamas afectaban a varios pisos del reactor $\mathrm{N}^{\mathrm{o}} 4$ y se acercaban peligrosamente al edificio donde se encontraba el reactor $\mathrm{N}^{0} 3$. El comportamiento de los bomberos durante las tres primeras horas del accidente fue fundamental al evitar que el fuego se extendiera al resto de la planta. Aún así, pidieron ayuda a los bomberos de Kiev, debido a la magnitud de la catástrofe. Los operadores de la planta llevaron a los otros tres reactores a parada fría. Dos días después, había 18 heridos muy graves y 156 heridos con lesiones medianas producidas por la radiación.

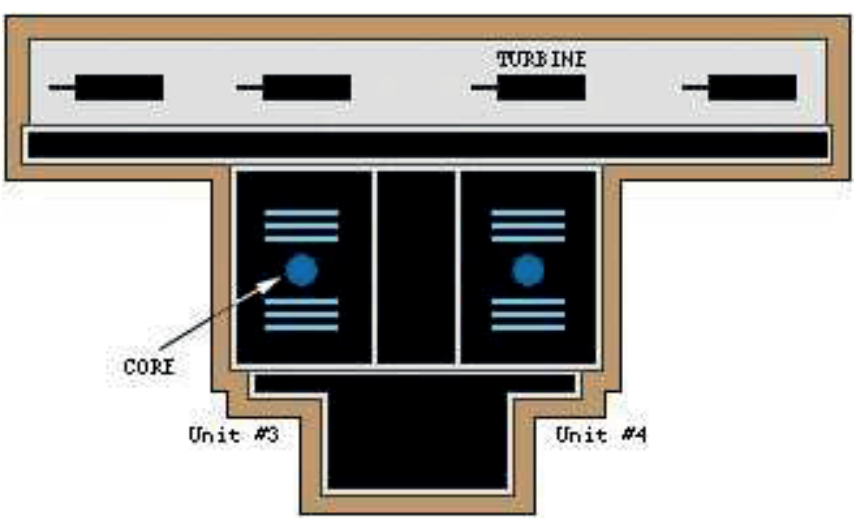

La mañana del sábado, varios helicópteros del ejército, comienzaron a arrojar sobre el núcleo una mezcla de materiales, consistente en: boro, como absorbente de neutrones, plomo, para contener la radiación, dolomita, para favorecer el enfriamiento, y arena y arcilla, como materiales aglutinantes. Cuando el 13 de mayo terminaron con este trabajo, se habían arrojado al núcleo unas 5.000 toneladas de estos materiales. 
Comenzó entonces la construcción de un túnel por debajo del reactor accidentado, para afianzar el terreno y evitar que toda la estructura superior, núcleo incluido, se hundiera, debido al peso de los materiales arrojados. En una semana se terminó y se inició el levantamiento de una estructura que envolviera al reactor aislándolo del exterior. El sensacionalismo que rodea a todo lo relacionado con la energía nuclear hizo que a esta estructura se la llamase sarcófago.

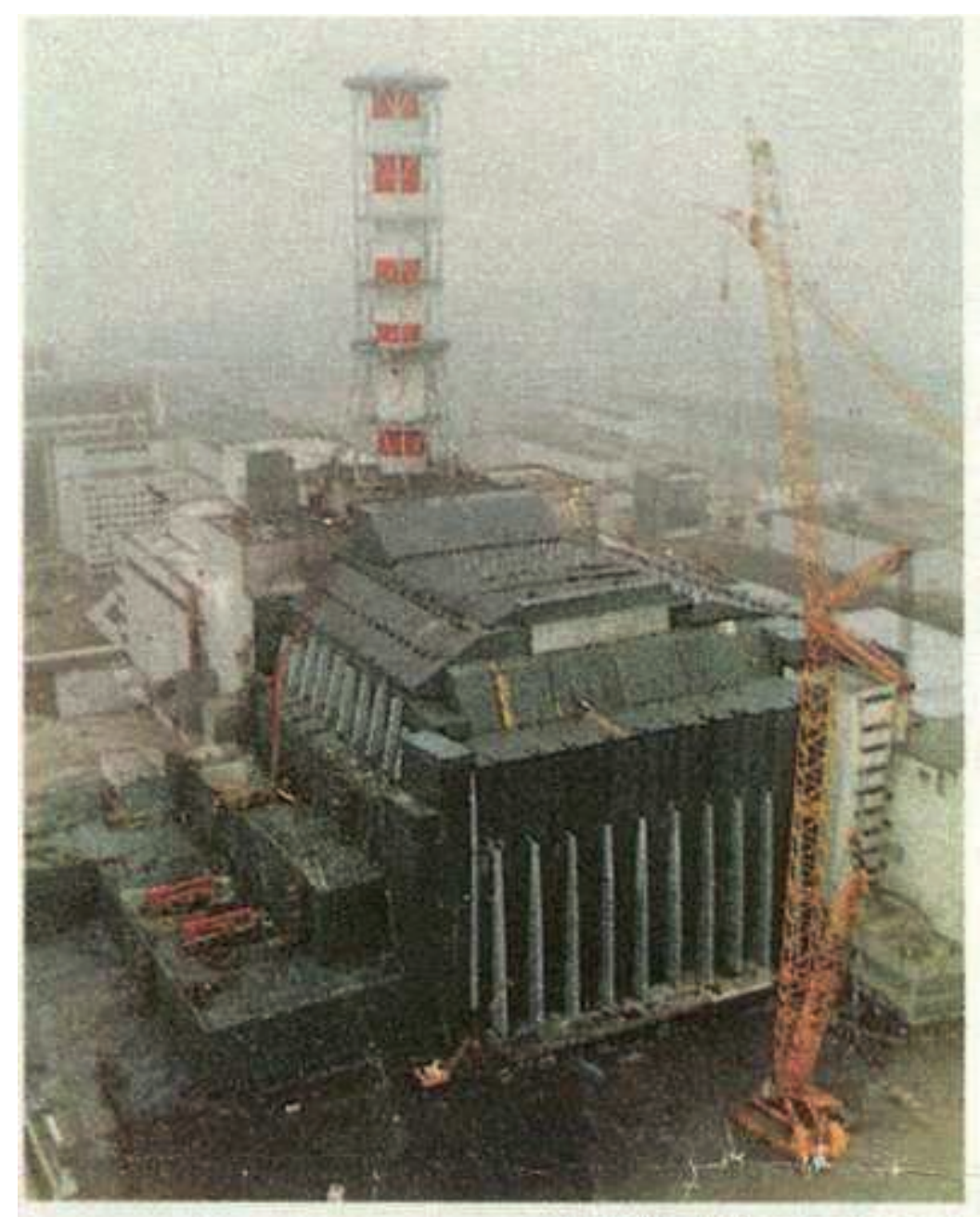

\section{Responsables de la catástrofe}


Y ahora viene la pregunta del millón ¿Por qué se hizo esta prueba que ya se había intentado en otras dos ocasiones $\mathrm{y}$, en las dos, hubo que abortar?

Lo más fácil es, simplemente, apelar a la ineptitud de los operadores; y así lo hicieron los responsables soviéticos en un primer momento, aunque esta explicación les duró poco. Es cierto que los operadores no tenían un entrenamiento aceptable, pero parece difícil pensar que no fueran conscientes de los riesgos de llevar a un reactor a una operación en estado inestable. Mucho menos creíble es suponer que un operador, por inexperto o incompetente que sea, opere un reactor inestable con todas las protecciones bloqueadas.

Parece ser que estos reactores tenían ciertos problemas con la refrigeración del núcleo en caso de pérdida de energía exterior (la correspondiente a la alimentación de las bombas de circulación). Debido a ello, era fundamental la realización de esta prueba para ver hasta donde se podía mantener la refrigeración del núcleo durante el tiempo que necesitaban los sistemas de emergencia en entrar en funcionamiento. Parece ser que, por ciertos problemas (¿de calendario?), si esta prueba no se realizaba cuando estaba previsto, debería retrasarse en, aproximadamente, un año y, en la antigua Unión Soviética, cumplir los objetivos políticos era más importante que demostrar que una instalación funcionaba correctamente. En la sentencia del juicio que se siguió contra los responsables de la central de Chernobyl, se recoge textualmente el siguiente párrafo:

"El 31 de diciembre de 1983, a pesar de que aún no se habían realizado las pruebas necesarias en el reactor número 4, Bryukhanov (director de la central) firmó un acta en la que se aceptaba la entrega del complejo del reactor y se certificaba que los trabajos se habían completado. Entre 1982 y 1985 se llevaron a cabo pruebas con el turbogenerador en desaceleración, con la intención de poner a punto el 
funcionamiento de los sistemas de seguridad. Estas pruebas no tuvieron éxito y fueron incompletas".

Lo que yo supongo, y repito, yo supongo, es que los operadores recibieron la orden de realizar la prueba; orden enviada por alguien que desconocía totalmente el funcionamiento de un reactor nuclear (¿quizás un político?); y, en la antigua U.R.S.S., era muy complicado desobedecer.

Sólo hace falta ver el estado en que quedó la sala de control para imaginar que nadie, en su sano juicio y sin ningún tipo de presión, se jugase graciosamente su propia vida.

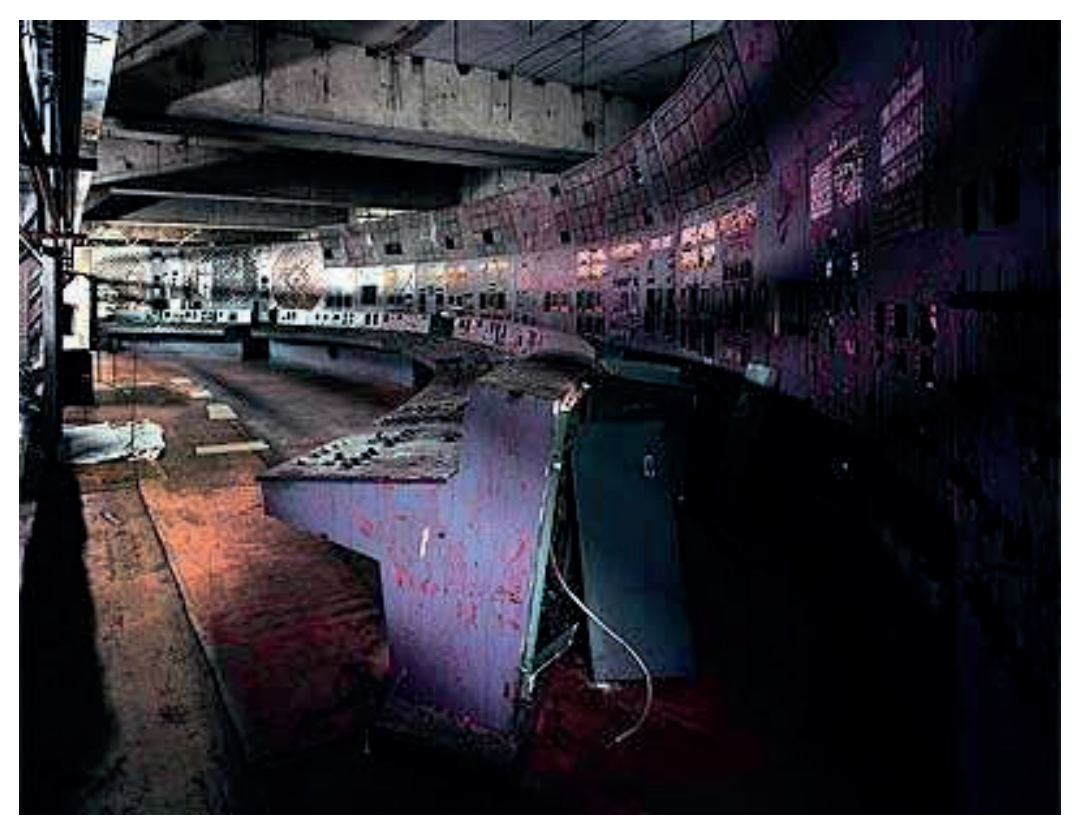

En cualquier caso, lo peor no fue el accidente en sí, sino el tratamiento que al mismo le dieron los incompetentes responsables de la emergencia.

\section{Consecuencias inmediatas del accidente}


Para darnos una idea de lo que ocurrió desde el punto de vista del control, o mejor dicho descontrol, de la emergencia, transcribo una parte de un artículo escrito por Jacques FROT en el año 2000:

...En los siguientes 10 días, alrededor de 12 exabequerels (exa = 1018) o 300 Mega curíes de isótopos radioactivos se liberaron a la atmósfera, contaminando significativamente un área de 150.000 kilómetros cuadrados (equivalente a 60.000 millas cuadradas - aproximadamente el área del estado de Iowa, como ejemplo en USA, o del conjunto de las Comunidades de Castilla y León, Cantabria, La Rioja y Aragón)NOTA_5 habitada por 6 millones de personas. También causó un incremento medible en el nivel de radiación ionizante en la mayor parte de Europa...

... Los efectos perjudiciales a la salud pública que siguieron a la explosión del reactor de Chernobyl no eran inevitables. Las únicas consecuencias inevitables eran la destrucción completa del reactor, la muerte de dos miembros del personal de operación que estabna encima del reactor en el momento que explotó y la contaminación radiactiva de una vasta superficie de territorio. Pero las circunstancias fueron tales que hubo efectos perjudiciales a la salud pública... 

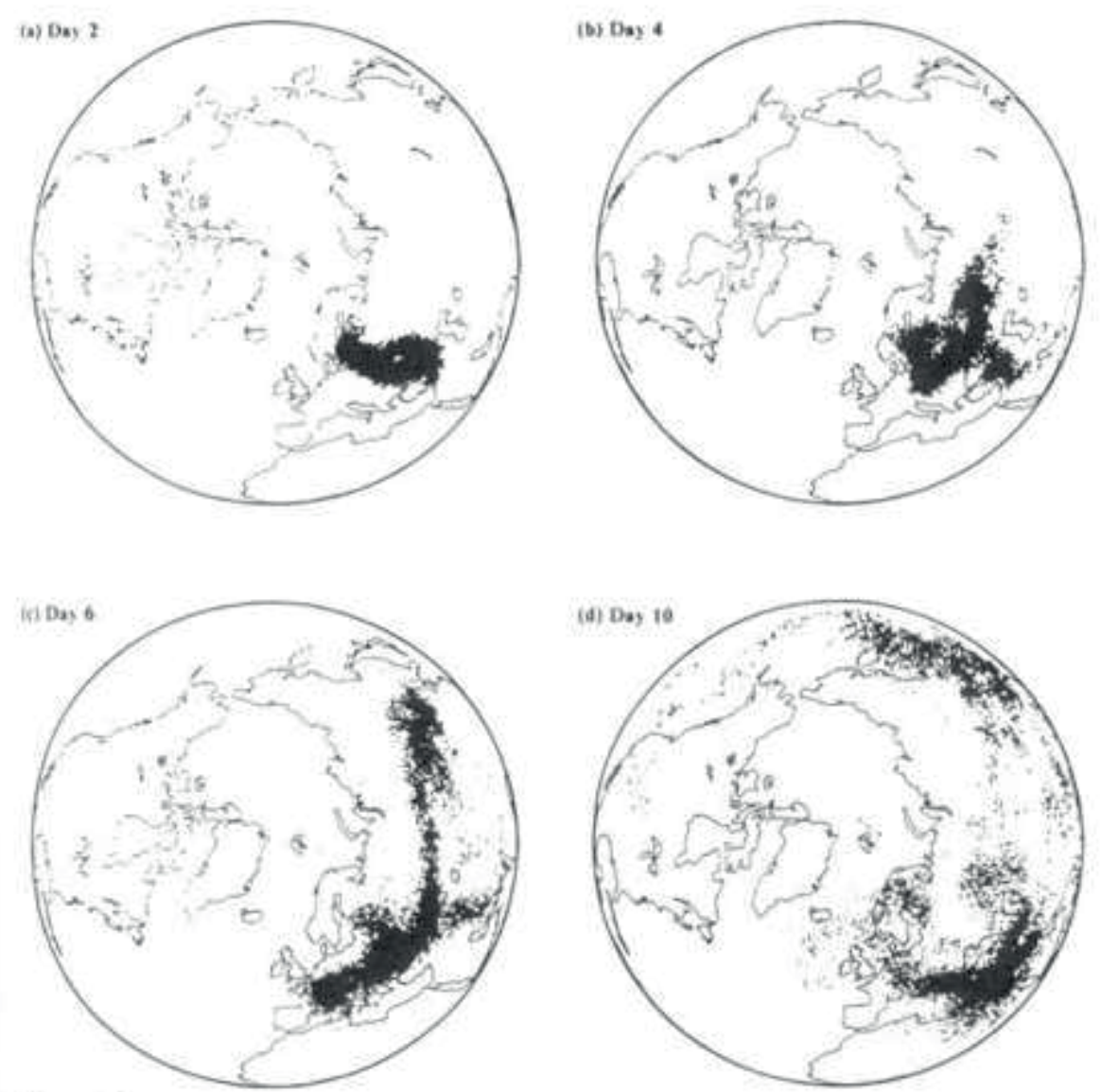

\section{Figure 15-6}

Spread of the radioactive plume from the Chemobyl accident over the Northem Hemisphere at (a) 2.

(b) 4, (c) 6, and (d) 10 days after the initial explosion. [Courtesy of Lawrence Livermore National

Laboratory. I

...Un plan de emergencia para radio-protección en caso de un accidente nuclear estaba desarrollado desde 1964. Incluía todas las medidas que se aceptan ahora universalmente: quedarse dentro de casa con ventanas y puertas cerradas, distribuir yodo estable, evacuar la población amenazada temporalmente, prohibir el consumo de comida probablemente contaminada, mover el ganado a pasturas no contaminadas, etc.

Cada acción estaba acompañada por criterios para el nivel de radioactividad que justificaba su aplicación. Este plan de la emergencia fue aprobado por el Ministerio de Salud de la URSS el 18 de diciembre de 1970, más de quince años antes de Chernobyl, pero se mantuvo como carta muerta. Un nuevo plan se presentó en 1985 
pero fue rechazado por el Ministro de Ingeniería Nuclear en septiembre, sólo siete meses antes del accidente. iiiLa razón dada era que un accidente de semejante naturaleza que requiriera estas medidas era "imposible en la URSS"!!!...

...En ausencia de un plan de emergencias como el francés "ORSEC" o PPI (ver nota al final de estos párrafos transcritos), las siguientes simples y elementales precauciones no se pusieron en efecto alrededor de Chernobyl, o se pusieron con retraso:

La transmisión inmediata de las noticias, incluso las instrucciones para quedarse dentro de casa con ventanas y puertas cerradas (esto no se hizo hasta que habían pasado 36 horas);

La prohibición en el consumo de leche fresca (después de 7 días);

La prohibición en el consumo de frutas frescas y verduras producidas localmente (después de 7 días);

La distribución inmediata de yodo estable (cápsulas de sodio o yoduro de potasio) con instrucciones para tragarlo inmediatamente (la oferta de EE.UU. fue rechazada);

La provisión inmediata de ropa de protección y respiradores a los bomberos, personal de operación y obreros de la recuperación (por mucho tiempo indisponible).

Durante las primeras semanas, el Iodo-131 radiactivo con vida media de 8 días fue la fuente principal de irradiación, y en el curso de los años siguientes ha causado varios casos de cáncer tiroideo. El yodo estable tragado sirve para saturar la glándula tiroidea inmediatamente y así prevenir la captación de Iodo-131 radiactivo... 
...Las autoridades civiles locales o no sabían nada o no le prestaron ninguna atención a esto. La ignorancia y falta de preparación eran tan profundas que, en los momentos que siguieron a la explosión, la inmensa mayoría de los actores en el drama: los grupos de operación del reactor, los directores de la central de potencia, las autoridades locales y superiores estuvieron tan aturdidos que fueron incapaces de apreciar la dimensión del desastre, incapaces en definir prioridades e incapaces incluso para emprender las actividades urgentemente requeridas.

Nota:

ORSEC es el acrónimo francés de la "Organización Regional para Asistencia." Es un plan detallado, región por región, para el rescate y las medidas de seguridad que deben ser activadas siempre que una emergencia natural de gran escala o de cualquier otro tipo ponga en riesgo la vida o las propiedades - inundaciones, tormentas, avalanchas, accidentes industriales, etc. ORSEC-Rad se relaciona con accidentes que pueden generar daños por irradiaciones. PPI es un suplemento de ORSEC y de ORSEC-Rad.

Estos planes son similares en todos los países occidentales con energía nuclear. En concreto en España cada central nuclear tiene desarrollados los procedimientos: PEI (Plan de Emergencia Interior), PVRE (Plan de Vigilancia Radiológica Exterior) además de otros manuales y procedimientos para el cálculo de dosis, operaciones de emergencia etc.

\section{Conclusión}


Así fue que algunos de los obreros de rescate, principalmente los bomberos en la central de potencia, fueron irradiados fatalmente porque trabajaron demasiado tiempo en áreas de alta contaminación sin equipo de protección adecuado y aun sin dosímetros. Veintiocho de ellos murieron. Estos 28 sacrificios pudieron evitarse.

Así fue que la población de Pripyat, alejada de 3 a 5 kilómetros, no fue informada ni evacuaas hasta la tarde del 27 de abril, más de 36 horas después de la explosión.

Así fue que no se distribuyeron tabletas de yoduro de potasio a los habitantes de la zona contaminada, o era demasiado tarde cuando fueron distribuidas para ser eficaces. Esas tabletas habrían protegido sus glándulas tiroideas de la irradiación por radio-yodo y así habrían evitado el cáncer. Es digno de mencionar que el yodo estable, de hecho, fue distribuido en la Polonia vecina y, como resultado, ese país no ha tenido exceso de cánceres de tiroides juveniles, aunque ciertas áreas recibieron una precipitación fuertemente radiactiva.

Así fue que la oferta de los EE.UU. el 1 de mayo, cinco días después de la explosión, de enviar una gran cantidad de yodo estable en forma de tabletas de yoduro de sodio fue rechazada.

Así fue que casi todos los 1.800 casos de cáncer tiroideo descubiertos a la fecha pudieron evitarse; probablemente se debieron al radio-iodo-131 enviado a la atmósfera por la explosión y por el fuego que siguió.

Así fue que el 2 de mayo, siete días después de la explosión, el consumo de productos agrícolas locales fue prohibido.

Así fue que, durante la primavera de 1986, finalmente, se evacuaron 120.000 personas, mientras que, por falta de instrumentos de medición, y por desconocimiento de los adelantos en radio-biología y radio-protección desarrollado 
por científicos en la URSS y en otras partes, la comunidad médica y las autoridades soviéticas no tenían la seguridad de que estas evacuaciones estavieran justificadas.

Así fue que la población mal informada y desinformada cayó presa del pánico, y pronto comprendió que las autoridades públicas habían perdido el control de la situación.

Así fue que las personas se convirtieron en víctimas de cuentos y rumores, siendo aun hoy el pan de los "mercaderes del miedo", quienes habitan en la prensa local, regional, nacional e internacional.

Así fue que muchos de los obreros de la recuperación y los evacuados cayeron victimas de la tensión psicológica; además de muchos suicidios, el trauma psicológico les llevó a enfermedades respiratorias, digestivas y cardio-vasculares. Estos casos no son el resultado directo de la irradiación, pero constituyen, por mucho, los más grandes efectos perjudiciales para la salud pública infligida por la explosión de Chernobyl.

Así fue que el contexto político del accidente de Chernobyl hizo imposible evitar una cantidad considerable de efectos perjudiciales para la salud pública; esto a pesar que el conocimiento médico y las técnicas preventivas y curativas habían existido en la Unión Soviética durante años y años.

Aquí de nuevo, se puede decir bien que los aspectos de salud del evento de Chernobyl fueron mucho más un evento soviético que un evento nuclear....

Actualmente los reactores RBMK están todos parados salvo algunos (creo que sólo tres) que, por razones puntuales de necesidades energéticas, siguen en operación. A estos reactores se les han realizado diversas modificaciones para corregir sus defectos de diseño. 
Y por último, pasad por el enlace que os dejo; ya me diréis que os parece.

http:/ / www.astroseti.org/vernew.php?codigo=2152\&barra=off

\section{Notas de VA}

Nota 1.- Mw es la abreviatura de Megavatio, es decir, un millón de vatios, siendo el vatio la unidad de potencia.

Nota 2.- Las barras de control están formadas por un material que absorbe los neutrones totalmente. Así, si se introducen las barras de control entre las barras de combustible y de moderador, la absorción de neutrones hace que las reacciones de fusión se den en menor número. El número total de barras se calcula para poder parar el reactor completamente. Dependiendo del número de barras introducidas, la potencia del reactor cambiará. De esta forma se puede regular la potencia del reactor.

Nota 3.- El agua que refrigera directamente el núcleo del reactor está en contacto con los materiales radiactivos y, por tanto, está altamente contaminada. Este circuito recibe el nombre de primario.

Por otra parte, en el tipo de reactor que nos ocupa, de agua en ebullición, el vapor producido en el reactor (circuito primario) pasa directamente a las turbinas. El problema derivado de esa configuración consistió en que, al tener la temperatura tan alta y la presión tan baja (relativamente hablando), estaban muy cerca de la saturación, por lo que, ya desde la parte inferior del núcleo se producían burbujas, es decir, tenían mucha cantidad de huecos. 
Nota 4.- El yodo puede encontrarse en el aire, el agua y el suelo de forma natural. Las fuentes más importantes de yodo natural son los océanos. El yodo en el aire se puede combinar con partículas de agua y precipitar en el agua o los suelos. El yodo en los suelos se combina con materia orgánica y permanece en el mismo sitio por mucho tiempo. Las plantas que crecen en estos suelos pueden absorber yodo. El ganado y otros animales absorberán yodo cuando coman esas plantas.

Este yodo pasará posteriormente a los humanos consumidores de estos productos naturales (carne, verduras, frutas y, fundamentalmente, leche) y se fija, sobre todo, en la glándula tiroides.

El yodo en las aguas superficiales se evaporará y volverá a entrar en el aire.

El yodo puede ser radioactivo. Los isótopos radioactivos se forman de manera natural durante reacciones químicas en la atmósfera. La mayoría de los isótopos radioactivos del yodo tienen unas vidas medias muy cortas y se transforman rápidamente en compuestos estables de yodo. Sin embargo, hay una forma radioactiva del yodo, el yodo 131, que tiene una vida media de 8 días y que es, por tanto, seriamente perjudicial para el medio ambiente. Este isótopo entra en el aire desde las plantas de energía nuclear, donde se forma durante el procesamiento del uranio y el plutonio. Los accidentes en las plantas nucleares han provocado la emisión de grandes cantidades de yodo 131 inyectadas en la atmósfera.

En un accidente como el que nos ocupa el Yodo 131 es la principal fuente de irradiación para los humanos. En estos casos, se deben suministrar grandes cantidades de Yodo estable, no radiactivo, a la población, para que éste sature la concentración de yodo en la glándula Tiroides y no se pueda fijar el Yodo radiactivo 
Nota 5.- El Curie (Ci) es una antigua medida que mide la actividad de una muestra radiactiva. Equivale a la actividad de un gramo de Radio y se define como:

1 Curie $=$ la cantidad de un material radiactivo dado que producirá $3,7 \times 1010$ desintegraciones nucleares por segundo, es decir, treinta y siete mil millones de desintegraciones por segundo.

El Becquerel (Bq) es la unidad más reciente del Sistema Internacional para medir la actividad y se define como:

1 Becquerel = la cantidad de un material radiactivo dado que producirá una desintegración nuclear por segundo.

Por consiguiente:

$1 \mathrm{Ci}=3,7 \times 1010 \mathrm{~Bq}$

Exa es el prefijo del Sistema Internacional que indica una cantidad equivalente a 1018, es decir, un trillón. En nuestro caso, 12 exabequerels corresponden a doce trillones de Becquerels o, equivalente y aproximadamente, trescientos millones Curies. 